\title{
The importance of molecular cytogenetic analysis prior to using cell lines in research: The case of the KG-1a leukemia cell line
}

\author{
FRANCA PELLICCIA $^{1}$, VALENTINA UBERTINI ${ }^{1}$ and NAZARIO BOSCO ${ }^{2}$ \\ ${ }^{1}$ Department of Biology and Biotechnology, Sapienza University, I-00185 Rome, Italy; \\ ${ }^{2}$ The Rockefeller University, New York, NY 10065, USA
}

Received January 30, 2012; Accepted April 18, 2012

DOI: 10.3892/ol.2012.709

\begin{abstract}
KG-1 and its less differentiated subline KG-1a are leukemia cell lines used in research in a number of laboratories. The karyotypes of the two lines were initially identical. In the following years, further analysis revealed that the cell lines had acquired additional karyotypical abnormalities and differed in the presence of certain typical chromosomal rearrangements. To obtain cytogenetic authentication prior to the use of the two cell lines, we analyzed their karyotype by combining DAPI- and CMA-chromosome bandings and a fluorescence in situ hybridization (FISH)-based approach by using BAC clones useful for the identification of chromosome regions of interest. Sequences of the $M Y C, P L Z F, R A R A$ and $B C R$ genes, that are known to play a critical role in leukemogenesis, and certain BAC clones mapped to five known common fragile sites (CFS) were used for the FISH analysis. A telomeric probe (TTAGGG)n and a set of BAC clones were used to characterize the marker chromosome der(1) that was observed in the cell line KG-1a. The existence of notable differences between the karyotype of the KG-1a cell line previously described, and that described in this study, demonstrate that the use of established cancer cell lines should be preceded by cytogenetic and/ or molecular characterization.
\end{abstract}

\section{Introduction}

KG-1 and KG-1a leukemia cell lines have been used in research in numerous laboratories. KG-1, a cell line established in 1978 by Koeffler and Golde (1), was derived from the bone marrow of a male individual with erythroleukemia that developed into acute myeloid leukemia (AML). The KG-1 cells are mostly at the myeloblast or promyelocyte stage of maturation.

KG-1a is a less differentiated subline of KG-1 developed after 35 passages. The karyotypes of the two lines were

Correspondence to: Professor Franca Pelliccia, Dipartimento di Biologia e Biotecnologie, Università La Sapienza, Piazzale Aldo Moro, 5, I-00185 Rome, Italy

E-mail: franca.pelliccia@uniroma1.it

Key words: KG-1a, leukemia cell line initially identical (2). The comparative karyotypic analysis of the two cell lines performed in subsequent years by other authors using various cytogenetic and molecular techniques $(3,4)$ revealed that the cell lines had acquired additional karyotypical abnormalities and, although sharing several identical structural aberrations, differed in the presence of certain typical chromosomal rearrangements. Particularly, Mrózek et al (4) used G-banding, spectral karyotyping (SKY) and fluorescence in situ hybridization (FISH) analyses to produce a detailed description of chromosome aberrations in the KG-1 and KG-1a cell lines. Comparative analysis of the two karyotypes is extremely useful for authentication of the two cell lines.

To obtain a characterization and cytogenetic authentication of the two cell lines prior to use, their karyotype was analyzed by combining DAPI- and CMA-chromosome bandings and a FISH-based approach. For FISH analyses a number of BAC clones useful for the identification of chromosome regions of interest were employed, including the $M Y C$, retinoic acid receptor $(R A R A)$, promyelocytic leukemia zinc finger $(P L Z F)$ and breakpoint cluster region $(B C R)$ genes. Evidence suggests that the $M Y C$ copy number gain has a role in human myeloid leukemia (5 and refs. cited therein). The RARA, PLZF and BCR genes are known to be involved in translocations generating fusion proteins that play a critical role in leukemogenesis (6). A number of BAC clones mapped to five known common fragile sites (CFS) were also used. CFS are preferential loci for double strand DNA breaks under stressful growing cell conditions. In cancer cells, CFS are frequently involved in recurrent chromosome rearrangements (7). A number of BAC clones mapped on chromosome 1 were used to characterize the marker chromosome der(1) that we observed in the cell line KG-1a. This study reveals the results of our analysis.

\section{Materials and methods}

Cell lines. The analyzed KG-1 and KG-1a cell lines were obtained from the American Type Culture Collection (Manassas, VA, USA) approximately 20 years ago. The cells had not been maintained in culture for long periods of time but were refreshed a certain number of times. The cells were cultured in RPMI-1640 with $2 \mathrm{mM} \mathrm{L-glutamine,} 10 \% \mathrm{FCS}$ at $37^{\circ} \mathrm{C}$ in $5 \% \mathrm{CO}_{2}$. 

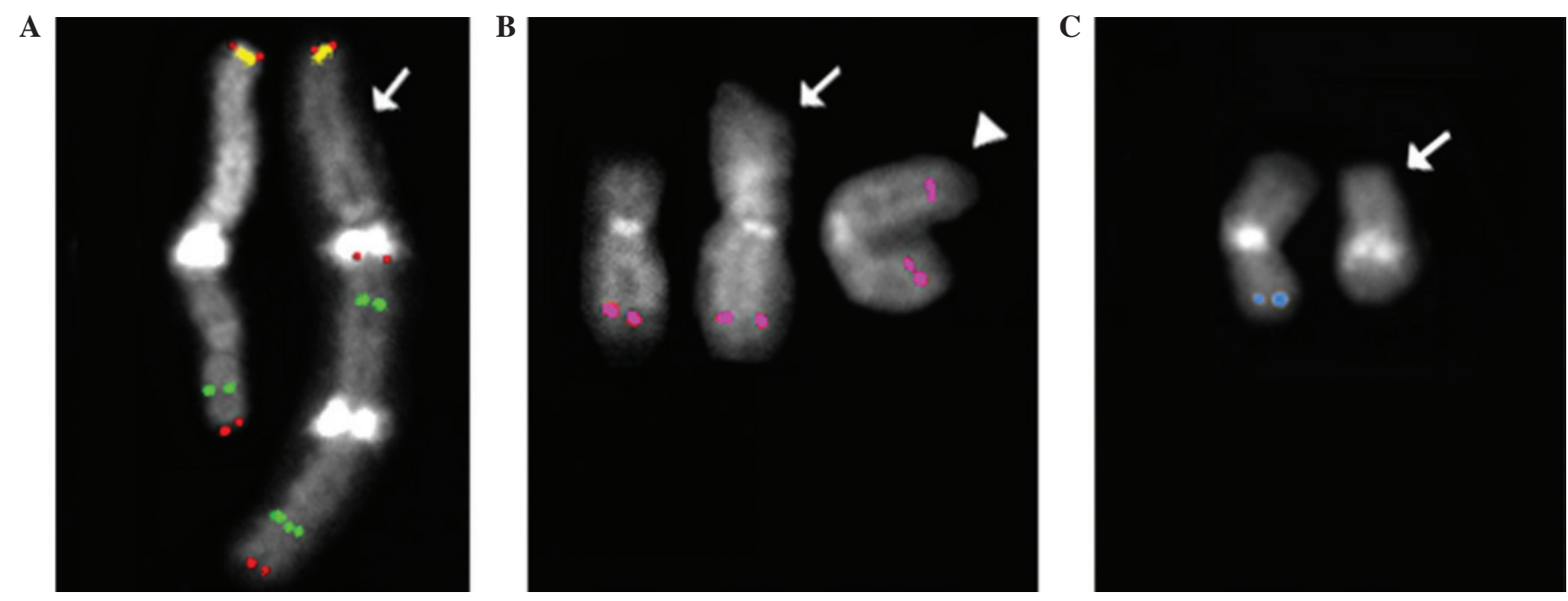

Figure 1. FISH experiments on chromosomes from KG-1a cells. (A) Chromosomes 1 and der(1) (arrow) are hybridized by a telomeric probe (red), BAC clones RP11-319A11 (yellow) and RP11-434B7 (green). The palindromic arrangement of the last clone and the interchromosomal telomeric signal can be detected on the chromosome der(1). (B) Chromosomes 8, der(8)t $(8 ; 12)$ (arrow) and idic(8)(p11) (arrowhead) are hybridized by the BAC clone RP11-440N18 (MYC). (C) Chromosomes 16 and del(16) are hybridized by the BAC clone RP11-264L1 (FRA16D). FISH, fluorescence in situ hybridization.

Chromosome analysis. Cell harvesting, DAPI- and CMA-chromosome bandings were performed using standard methods. The BAC clones used for the FISH analysis were: RP11-440N18 mapped at 8q24.21 that contains the entire MYC gene; RP11-1006G20 (11q23.2), the majority of the $P L Z F$ gene; RP11-1152A10 (17q21.2), the entire RARA gene; and RP11-434O9 (22q11.23), the first part of the $B C R$ gene. BAC clones mapped to five known CFS were also used: RP11-158L8 (FRA2G, 2q31), RP11-48E21 (FRA3B, 3p14.2), RP11-425P5 (FRA7B, 7p22.1), RP11-36B6 (FRA7H, 7q32.3) and RP11-264L1 (FRA16D, 16q23.2). We also used a telomeric probe (TTAGGG)n, generated by PCR (8) and a set of BAC clones mapped onto chromosome 1 to characterize the marker chromosome der(1) that was observed in the cell line KG-1a: RP11-319A11 (1p36.32), RP11-79E5 (1q12), RP11-434B7 (1q32.3), RP11-385F5 (1q43) and RP11-438F14 (1q44). The BAC stabs were obtained from CHORI (Oakland, CA, USA), and a number of them were supplied by the Cytogenetic Unit of the University of Bari (Italy). FISH experiments were performed as described in a previous study (9).

\section{Results}

KG-1 cell line karyotype. The karyotype of the KG-1 and KG-1a cell lines revealed a pseudodiploid modal number of chromosomes. The karyotype of the KG-1 cell line analyzed was the same as that described by Mrózek et al (4): $46, \operatorname{der}(4) \mathrm{t}(4 ; 8)(\mathrm{q} 31 ; \mathrm{p} 21),-5, \operatorname{del}(7)(\mathrm{q} 22 \mathrm{q} 35), \operatorname{der}(8) \mathrm{t}(8 ; 12)$ $(\mathrm{p} 11 ; \mathrm{q} 13),+\mathrm{idic}(8)(\mathrm{p} 11) \times 2,-12, \operatorname{der}(17)(5 \mathrm{pter} \rightarrow 5 \mathrm{p} 11:: 5 \mathrm{q} 1$ $3 \rightarrow 5 q 31:: 17$ p11.2 $\rightarrow$ cen $\rightarrow 17 q$ ter $), \operatorname{der}(20) t(12 ; 20)(? ; p 13)$.

Therefore the $M Y C$ gene was present in six copies: one on a normal chromosome 8 , four on the two copies of the idic(8) (p11) and one on the der(8)t $(8 ; 12)$; the PLZF and BCR genes were present in the normal number and chromosomal location and the RARA gene was present in two copies: one localized on a normal chromosome 17 , the other on the short arm of the $\operatorname{der}(17) t(5 ; 17)$.
KG-1a cell line karyotype. Differing from KG-1, the karyotype of the KG-1a cell line showed specific differences from that described by Mrózek et al (4): the presence, besides a normal chromosome 1 , of a chromosome der(1) that has the duplication of the entire long arm (Fig. 1A); chromosome $\operatorname{der}(8 ; 12)$ (Fig. 1B, arrow) instead of the der(8;22); only one normal copy of the chromosome 11 besides the i(11)(q10); a deleted chromosome 16q (Fig. 1C); two normal copies of chromosome 22 and lack of the chromosome Y. Accordingly, the description of the KG-1a karyotype observed (Fig. 2) is: $45-47, X,-Y, \operatorname{der}(1)($ pter $\rightarrow$ cen $\rightarrow$ q12 $:: q$ ter $\rightarrow$ q12::q12 $\rightarrow$ qter $)$, $\operatorname{der}(4) t(4 ; 8)(\mathrm{q} 31 ; \mathrm{p} 21),-5, \operatorname{del}(7)(\mathrm{q} 22 \mathrm{q} 35), \operatorname{der}(8) \mathrm{t}(8 ; 12)(\mathrm{p} 11 ; \mathrm{q} 13)$, $+\mathrm{idic}(8)(\mathrm{p} 11), \mathrm{i}(11)(\mathrm{q} 10),-12, \operatorname{del}(16)(\mathrm{q} 13 \sim 21), \operatorname{der}(17)(5 \mathrm{pter} \rightarrow$ $5 \mathrm{p} 11:: 5 \mathrm{q} 13 \rightarrow 5 \mathrm{q} 31:: 17 \mathrm{p} 11.2 \rightarrow$ cen $\rightarrow 17 \mathrm{qter}),+\operatorname{der}(19) \mathrm{t}(14 ; 19)$ (q13;q13.4),der(20)t(12;20)(?;p13).

Therefore, in the KG-1a karyotype the MYC gene was present in four copies: one on a normal chromosome 8 , two on the $\operatorname{idic}(8)(\mathrm{p} 11)$ and one on the $\operatorname{der}(8) \mathrm{t}(8 ; 12)$ (Fig. 1B). We also observed that the PLZF gene was present in three copies: one on a normal chromosome 11 and two on the i(11) (q10). The RARA gene was present in two copies: one localized on a normal chromosome 17 , the other on the short arm of the der(17)t $(5 ; 17)$. The $B C R$ gene was present in normal number and chromosomal location on two chromosomes 22. Of note is that due to the presence of the chromosome der(1) bearing two copies of the long arm of the chromosome 1 , three copies of the 54 genes indicated as involved in cancer (10) localized on the long arm of this chromosome are present (Fig. 3).

\section{Discussion}

Koeffler et al (2) reported the presence of a large submetacentric chromosome (MAR-1) in the karyotype of the KG-1 and KG-1a cell lines, besides two normal copies of chromosome 1. Moreover, Furley et al (3) described the presence of a der(1) (mar 1) only partially identified and slightly 


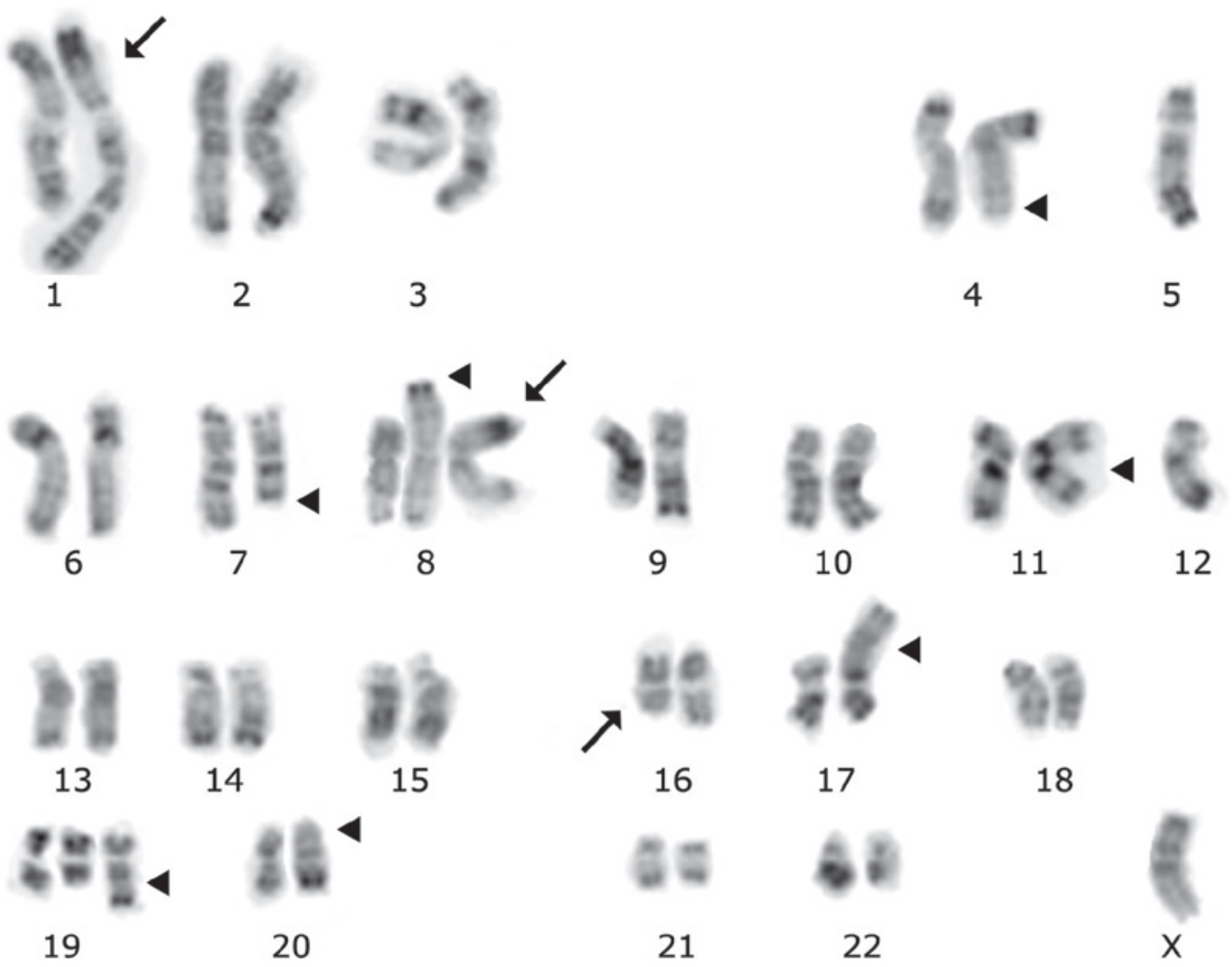

Figure 2. R-banding karyotype of the KG-1a cell line as mentioned in Materials and methods. The arrowheads indicate the chromosome rearrangements previously described by Mrózek et al (4). The arrows indicate the rearrangements only present in the KG-1a cell line described in the present study.

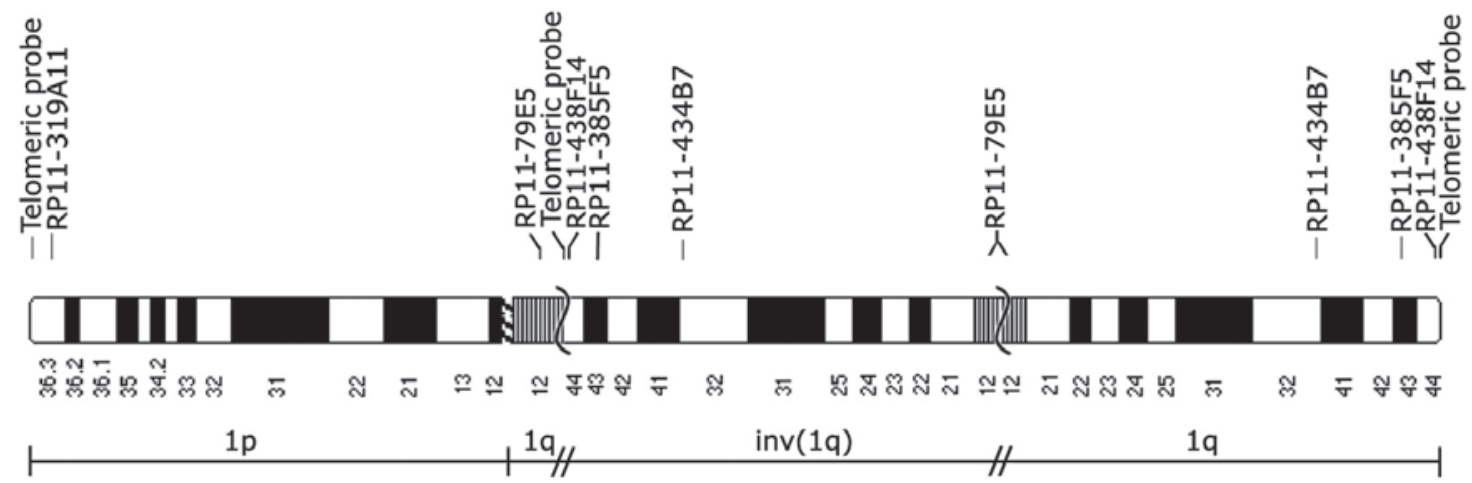

Figure 3. Map of the chromosome der(1) showing the BAC clones used as probes in the present study. The two curved lines indicate the breakpoint at the origin of the rearranged chromosome.

different in the two cell lines, besides two normal copies of chromosome 1 . Therefore, it is likely that the $\mathrm{KG}-1$ cell line examined by Mrózek et al (4) and by the authors of this study has lost the chromosome MAR-1 observed by Koeffler et al (2) and by Furley et al (3) and has maintained two copies of chromosome 1. Conversely, the KG-1a cell line examined by Mrózek et al (4) lost the chromosome MAR-1 and maintained the two copies of chromosome 1 , whereas the KG-1a cell line examined in the present study has lost one normal chromosome 1 and maintained the $\operatorname{der}(1)$ described in the present study.
The differences between the KG-1a karyotype described by Mrózek et al (4) and the one described in the present study demonstrate again that although the established cancer cell lines maintained in culture or refreshed numerous times in different laboratories derive from the same original cell lines, they may have different genotypic characteristics due either to the selection of subclones or to intervening mutations. Therefore, the use of established cancer cell lines for research purposes in cancer biology, and in any other field, should be preceded by a proper cytogenetic and molecular characterization (11). 


\section{References}

1. Koeffler HP and Golde DW: Acute myelogenous leukemia: a human cell line responsive to colony-stimulating activity. Science 200: 1153-1154, 1978

2. Koeffler HP, Billing R, Lusis AJ, et al: An undifferentiated variant derived from the human acute myelogenous leukemia cell line (KG-1). Blood 56: 265-273, 1980.

3. Furley AJ, Reeves BR, Mizutani S, et al: Divergent molecular phenotypes of KG1 and KG1a myeloid cell lines. Blood 68 1101-1107, 1986

4. Mrózek K, Tanner SM, Heinonen K and Bloomfield CD Molecular cytogenetic characterization of the KG-1 and KG-1a acute myeloid leukemia cell lines by use of spectral karyotyping and fluorescence in situ hybridization. Genes Chromosomes Cancer 38: 249-252, 2003.

5. Jones L, Wei G, Sevcikova S, et al: Gain of MYC underlies recurrent trisomy of the MYC chromosome in acute promye locytic leukemia. J Exp Med 207: 2581-2594, 2010.
6. Guidez F, Parks S, Wong H, et al: RARalpha-PLZF overcomes PLZF-mediated repression of CRABPI, contributing to retinoid resistance in $\mathrm{t}(11 ; 17)$ acute promyelocytic leukemia. Proc Natl Acad Sci USA 104: 18694-18699, 2007.

7. Arlt MF, Casper AM and Glover TW: Common fragile sites. Cytogenet Genome Res 100: 92-100, 2003.

8. Ijdo WJ, Wells RA, Baldini A and Reeders ST: Improved telomere detection using a telomere repeat probe (TTAGGG)n generated by PCR. Nucleic Acids Res 19: 4780, 1991.

9. Pelliccia F, Bosco N and Rocchi A: Breakages at common fragile sites set boundaries of amplified regions in two leukemia cell lines K562 - Molecular characterization of FRA2H and localization of a new CFS FRA2S. Cancer Lett 299: 37-44, 2010.

10. Dessen P, Knuutila S and Huret JL: Chromosome. Atlas Cytogenet Oncol Haematol, Jul 2004. Updated 2010. http:// AtlasGeneticsOncology.org.

11. Hughes P, Marshall D, Reid Y, et al: The costs of using unauthenticated, over-passaged cell lines: how much more data do we need? Biotechniques 43: 575-586, 2007. 